\title{
Integrating Social and Physical Sciences in a Graduate Student Workshop
}

Samuel J. Childs, Mariana G. Cains, and Faith P. Groff

\section{Quantifying and Communicating Uncertainty in High-Impact Weather Prediction}

What: Twenty-five select graduate students convened for a two-week workshop consisting of lectures from professionals as well as collaborative learning around the central theme of quantifying and communicating uncertainty in high-impact weather prediction.

When: $15-26$ July 2019

Where: Boulder, Colorado

AFFILIATIONS: Childs and Groff-Department of Atmospheric Science, Colorado State University, Fort Collins, Colorado; Cains-O’Neill School of Public and Environmental Affairs, Indiana University Bloomington, Bloomington, Indiana 


\section{The challenge of high-impact weather}

Meteorological disasters produce major impacts and loss of life across the United States each year. In 2018 alone, some 14 billion-dollar disasters were attributed to weather and climate phenomena (NOAA 2019). With each event comes numerous questions about how the longrange or short-range weather forecasts could have been more accurate, how the risks could have been more effectively communicated, and how societal and economic impacts could have been mitigated. In an era when computer models are continually getting faster and more versatile, machine-learning processes are emerging that can predict weather phenomena months in advance, and innovative observational techniques are employed to sample the atmosphere; the newest technology is always on the horizon. With these recent advancements within the weather and climate enterprises comes a growing need to be able to concisely and effectively communicate the latest research, as well as short-term forecasts of weather hazards, to the general public. A renewed energy for collaboration between physical and social scientists tends to emerge following large-scale disasters, but the obstacles and limitations toward healthy cross-disciplinary discussion tend to lessen the full potential of taking steps forward. While a disconnect between these two communities has been acknowledged, and multiple efforts have emerged in recent years to bridge that gap, one common hindrance to collaboration between those who conduct the science and those who communicate it has been gathering researchers and subject matter experts from both sides of the divide in the same room at the same time. The success of a recent multidisciplinary graduate student workshop proves that this roadblock can be overcome and provides hope as the next generation of scientists prepare to take on the challenges of communicating high-impact weather information to those who need it most.

\section{Interdisciplinary focus}

In July 2019, a select group of 25 graduate students convened at the National Center for Atmospheric Research (NCAR) in Boulder, Colorado, for a two-week colloquium administered by NCAR's Advanced Study Program (ASP: Fig. 1). Workshop organizers developed a

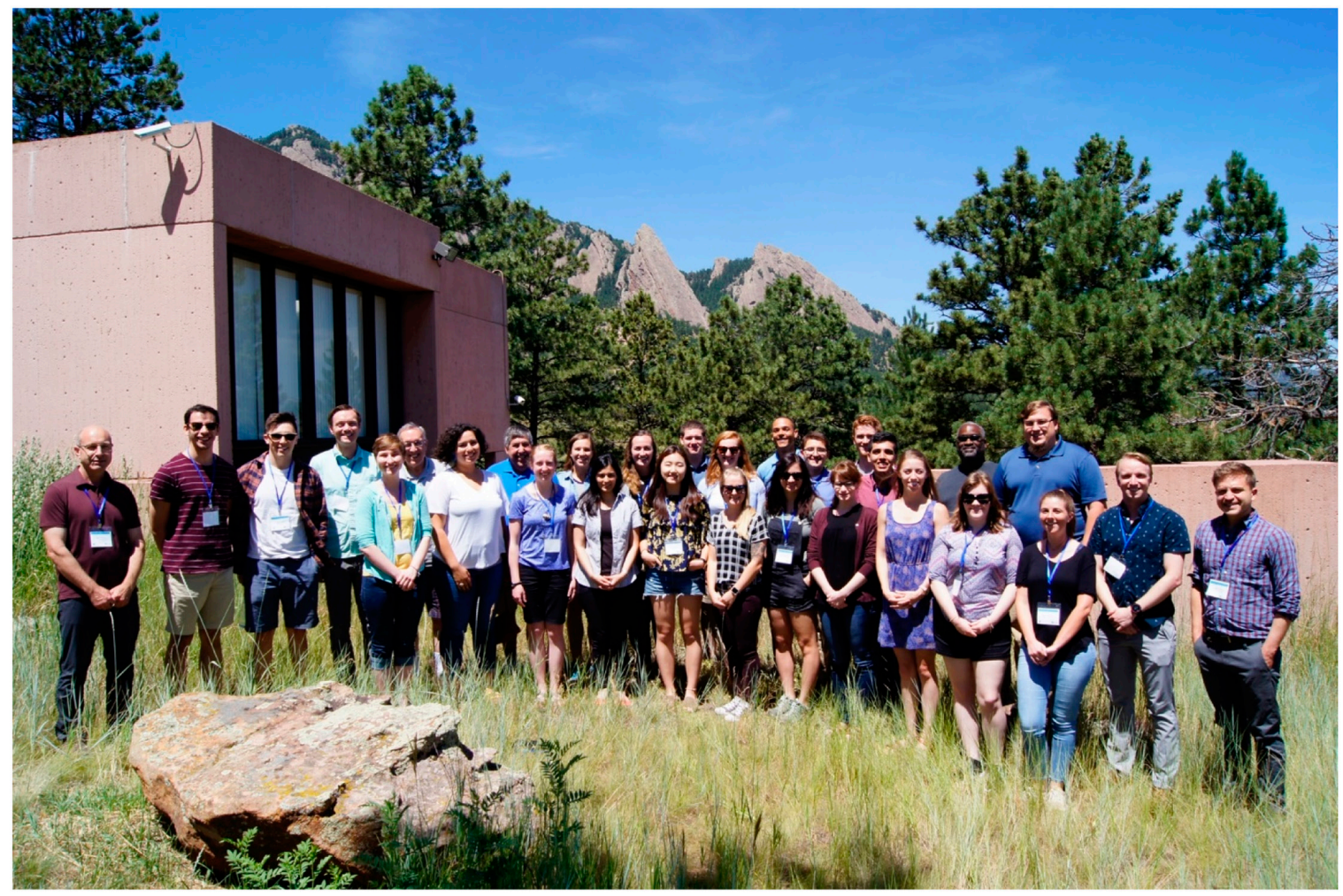

Fig. 1. Participants and organizing committee at NCAR Mesa Laboratory, Boulder, Colorado. Photo courtesy Scott Briggs, NCAR. 
unique theme for the 2019 installment of the colloquium, entitled "Quantifying and Communicating Uncertainty in High-Impact Weather Prediction.” This theme, which organizers admitted was hard to develop and execute, expressed the growing desire to bring together students from a variety of disciplines to promote the exchange of ideas within the specific context of high-impact weather events. Among the 25 participants were students from five different continents, whether by birth or via current study abroad appointments, at least four disciplines (Fig. 2), and more than 10 different majors within these disciplines, ranging from meteorology to disaster risk management, political science, and science communication. Such diversity among the group helped affirm the need for experts in a variety of fields to help solve weather and climate issues, and also fostered an environment for learning from those with very different

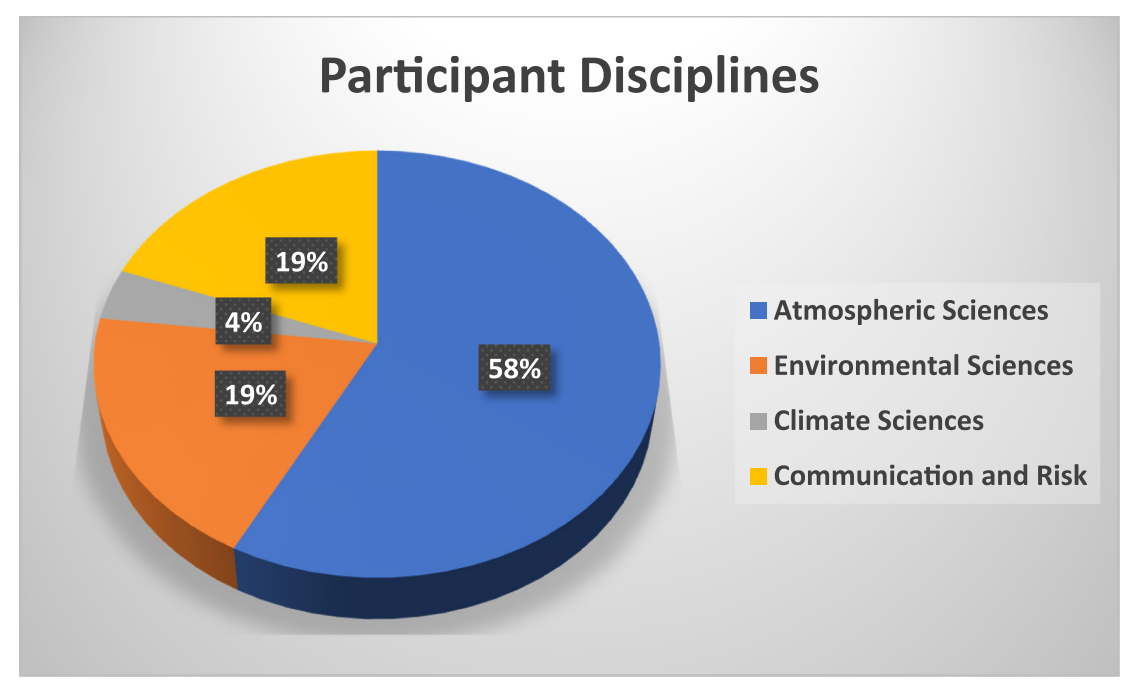

Fig. 2. Distribution of major fields of study of the colloquium participants. Each of these four broad fields can be subdivided into several individual majors. backgrounds and perspectives.

The workshop was structured as a series of daily lectures from academics and professionals from across the country, interwoven with professional development opportunities for the participants. Lectures were organized around three subthemes-predictability, machine learning, and risk communication-with multiple days showcasing each of these concepts. Given the interdisciplinary nature of the participants, speakers were asked to start their presentation with a brief autobiography, followed by a talk that was in the style of a collegiate lecture that highlighted the current state of knowledge as opposed to an in-depth analysis of recent research. Like the participants, speakers and presentations spanned a wide range of expertise, from highlighting state-of-the-art hail prediction using machine learning, to sharing how the private sector develops their apps with weather and climate forecasting tools that cater to the current consumer base, and promoting ethical and moral guidelines for conducting high-quality research. Figure 3 shows a photo of the typical lecture setup. Several speakers also led hands-on activities, giving participants a chance to utilize some of the latest technological advancements in the field. Numerous opportunities for networking and career development were also provided, including a networking dinner with local professionals in a variety of interdisciplinary positions, a resume/CV writing workshop in which students peer-reviewed the documents, and a briefing on how to talk with reporters.

\section{Collaboration in action}

Perhaps the most practical aspect of the workshop was a group project that spanned the two-week colloquium. Consistent with the overall theme, groups were assigned with at least one individual representing each subtheme of predictability, machine learning, and risk communication. Projects consisted of researching a recent high-impact weather event from multiple societal and meteorological angles, ultimately presenting recommendations for how the event's forecasting and/or communicative aspects could have been improved upon. Figure 4 shows a photo taken during one group presentation that highlighted forecasting challenges for a recent flood event in Ellicott City, Maryland. Projects were assessed by the 


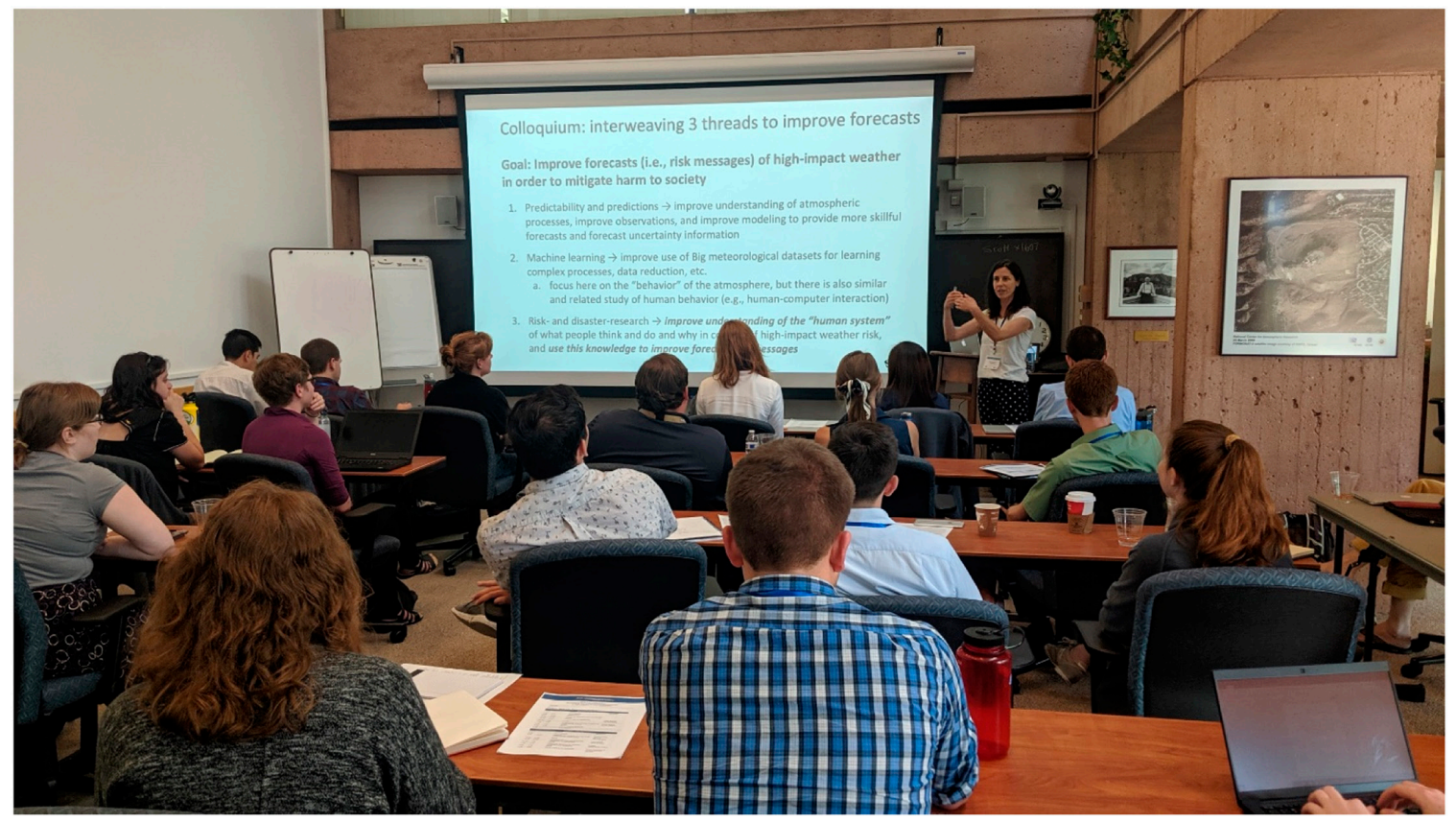

Fig. 3. Participants listen as organizing committee member Julie Demuth highlights the three components of improving forecasts of high-impact weather that would be the themes of the colloquium. Photo courtesy Russ Schumacher.

organizing committee, who gave constructive feedback and additional queries for future investigation. By integrating multiple perspectives into assessment of a single event, the students were able to practice working in diverse teams and also were exposed to the great benefit of combining expertise when trying to further the current research questions within the meteorological community.

Although much has been said regarding the challenges of bringing experts from social science, risk communication, atmospheric science, and computer science (to name a few),

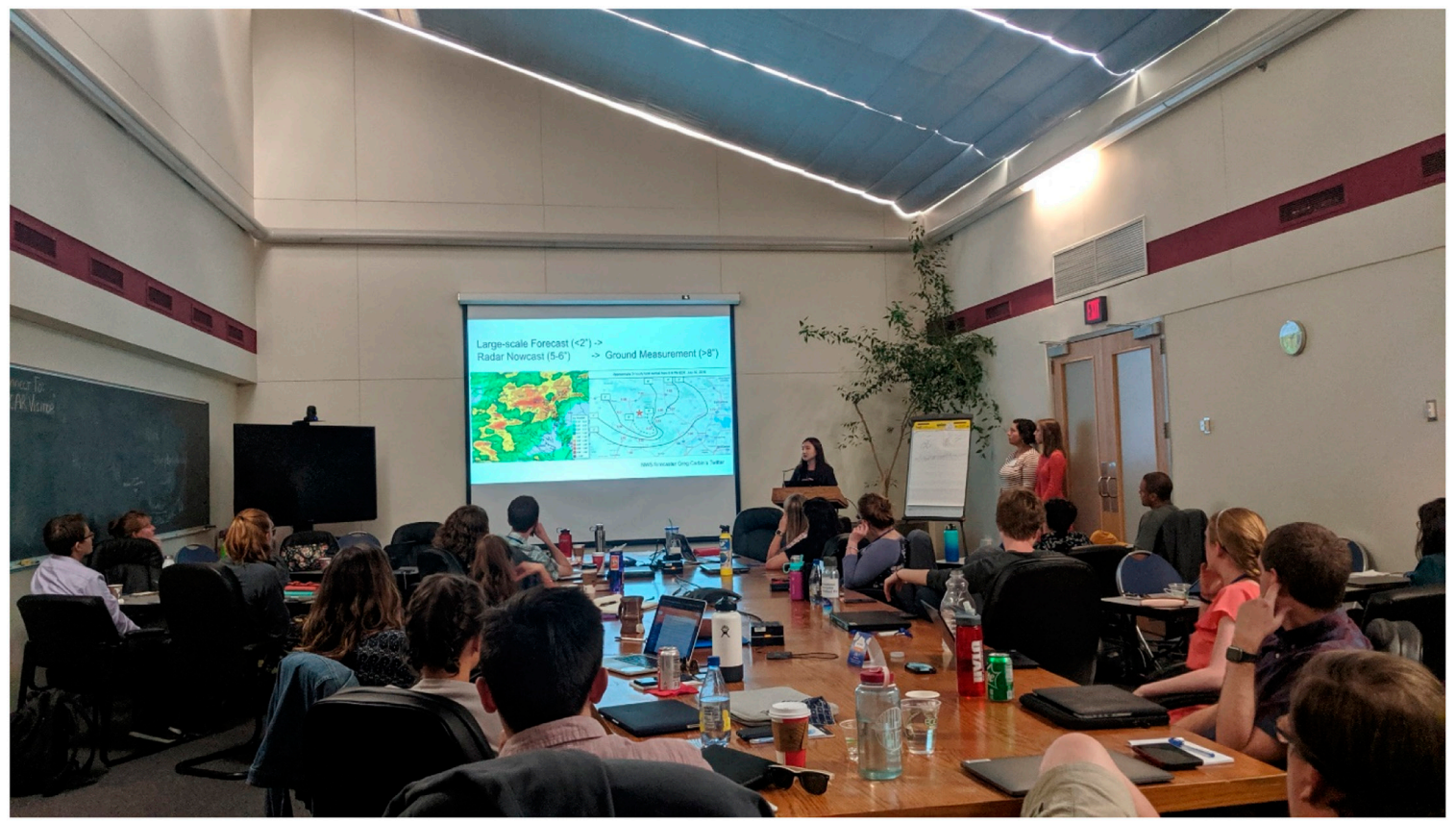

Fig. 4. One group presents their project findings during the last day of the colloquium. Photo courtesy Russ Schumacher. 
the 2019 NCAR ASP Colloquium was proof that it can be done. Workshop participant Michael Michaud concluded that while sustained efforts to improve predictability of high-impact weather events is vital, "the opportunity to come together with others to collaborate and share our passions is just as crucial.” Inspiring the up-and-coming generation of scientists to collaborate and communicate is an achievable task, and workshops such as this one should be replicated in other settings. Participant Austin Harris remarked that it was "rare to see a single topic treated from so many different perspectives, and it taught me that our jobs don't stop after the model run is finished or the forecast is issued." Indeed, a healthy balance of applauding the outstanding achievements in the weather predictability sphere and promoting integrated work with social scientists must be fostered for effective communication of high-impact weather threats and the ultimate protection of life and property. New ideas, collaborations, and friendships gained from this workshop will undoubtedly go a long way toward reaching those goals.

Acknowledgments. The authors wish to thank the 2019 NCAR ASP Colloquium organizing committee: Glen Romine, Rebecca Morss, Julie Demuth, and Chris Snyder (NCAR); Lance Bosart (University at Albany, State University of New York); and Russ Schumacher (Colorado State University). The National Center for Atmospheric Research is sponsored by the National Science Foundation.

\section{Reference}

NOAA, 2019: Billion dollar weather and climate disasters: Summary stats. NCDC, www.ncdc.noaa.gov/billions/summary-stats. 